\section{A case series of primary cutaneous B-cell lymphomas with atypical presentations: diagnostic and therapeutic challenges}

Primary cutaneous B-cell lymphomas (PCBCL) are defined as B-cell lymphomas of the skin without nodal, bone marrow, or visceral involvement at the time of diagnosis. ${ }^{1}$ They represent approximately $25 \%$ of primary cutaneous lymphomas. ${ }^{1,2}$ The histopathological diagnosis of PCBCL can be challenging in certain instances in which overlapping features are present. Nevertheless, identification of the correct subtype of PCBCL is imperative for determining the prognosis and avoiding inappropriate aggressive treatments which could lead to unnecessary morbidity. ${ }^{3}$ Here we present a series of three cases that highlight the distinguishing features between two subtypes of PCBCL.

The first case was a 57-year-old African American man with multiple pruritic nodules on his abdomen that appeared and rapidly progressed in size 6 months prior to presentation (Figure 1A). Review of systems was negative for pain, weight loss, night sweats or fever. Blood flow cytometry analysis was normal, and positron emission tomography (PET) scan was negative for metabolically active lymph nodes or systemic disease. Skin biopsy demonstrated sheets of large atypical lymphoid aggregates with centroblast morphology extending into the entire thickness of the dermis with an accompanying significant reactive small-sized lymphocytic infiltrate (Figure 1B). The large cells expressed CD20 and BCL6 but were negative for CD10, BCL2, and MUM-1 on immunostaining (Figure 1D-H). Ki67 stain showed a proliferation rate of $30-40 \%$. B-cell receptor clonality assay (ClonoSeq) identified two dominant immunoglobulin heavy chain sequences. An initial diagnosis of diffuse large B-cell lymphoma (DLBCL) was considered, but a complete histopathological review with clinical correlation led to a final diagnosis of primary cutaneous follicle center lymphoma (PCFCL) with diffuse pattern. Three large lesions were excised and local radiation therapy provided total regression of the rest of the abdominal tumors without recurrence to date (2 years).

The second case was an 83-year-old Caucasian male presenting with an erythematous, asymptomatic growth on the forehead that had increased in size to $6 \times 7 \mathrm{~cm}$ over 6 months (Figure 2A). The patient had no systemic symptoms of fever, night sweats, weight loss, or lymphadenopathy. Punch biopsy of the lesion demonstrated a dense lymphocytic neoplasm composed of centroblasts and immunoblasts positive for CD10, CD20, and BCL6 but negative for MUM1. Small-sized reactive lymphocytes were BCL2-positive (Figure 2B, D-H). Immunoglobulin heavy chain gene-rearrangement studies revealed a monoclonal population. Blood flow cytometry analysis and PET scan were negative for blood or systemic involvement. This case was also initially diagnosed as DLBCL, but a secondary histopathological review combined with clinical correlation led to a diagnosis of PCFCL with diffuse pattern. Radiation therapy provided complete regression of the tumor without recurrence to date (1.5 years).

The third case was a 72-year-old man with a history of chronic kidney disease and heart failure who presented with a tender pink tumor on his scalp that abruptly appeared as a small papule but rapidly grew in size to 4 x $4 \mathrm{~cm}$ over 1 month (Figure 3A). His clinical history was negative for fever, lymphadenopathy, fatigue, night sweats, and systemic symptoms. Punch biopsy revealed a sheet-like diffuse dense infiltrate composed of cells with immunoblastic morphology and high mitotic activity (Figure 3B). The atypical lymphocytes stained positive for CD10, CD20, BCL2, BCL6, and MUM1 (Figure 3D-H) with a more than $80 \%$ proliferative population based on Ki-67 positivity. Fluorescent in-situ hybridization (FISH) was positive for a $B C L 6$ gene rearrangement $(18 \%$ of nuclei) and negative for rearrangement of $M Y C, C C N D 1$, and $B C L 2$. A dominant immunoglobulin heavy chain sequence present in $99 \%$ of all nucleated cells was identified by immunosequencing (ClonoSeq). PET scan revealed a hypermetabolic scalp lesion with no evidence of lymph node or systemic involvement, and blood flow cytometry analysis was normal. The patient was diagnosed with primary cutaneous diffuse large B-cell lym-

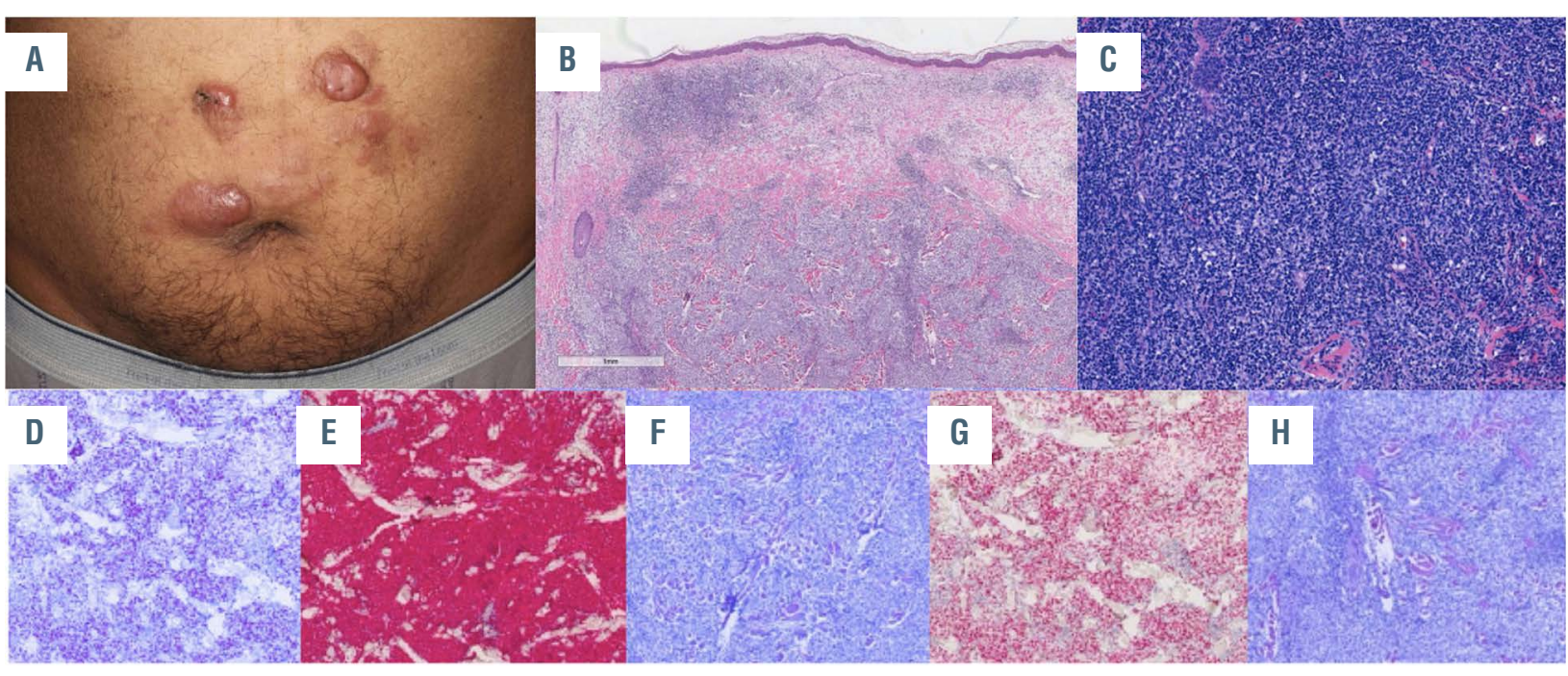

Figure 1. Primary cutaneous follicle center lymphoma identified by various features. (A) Three firm, ill-defined, pink tumors, 2 to $3 \mathrm{~cm}$ in size, with surrounding erythematous plaques and significant induration on the mid abdomen. (B, C) Dense lymphocytic infiltrate of small to medium sized lymphocytes with condensed nuclei (hematoxylin \& eosin: [B] 40x, [C] 100x) that are (D) CD10-negative, (E) CD20-positive, (F) BCL2-negative, (G) BCL6-positive, and (H) MUM1-negative. 


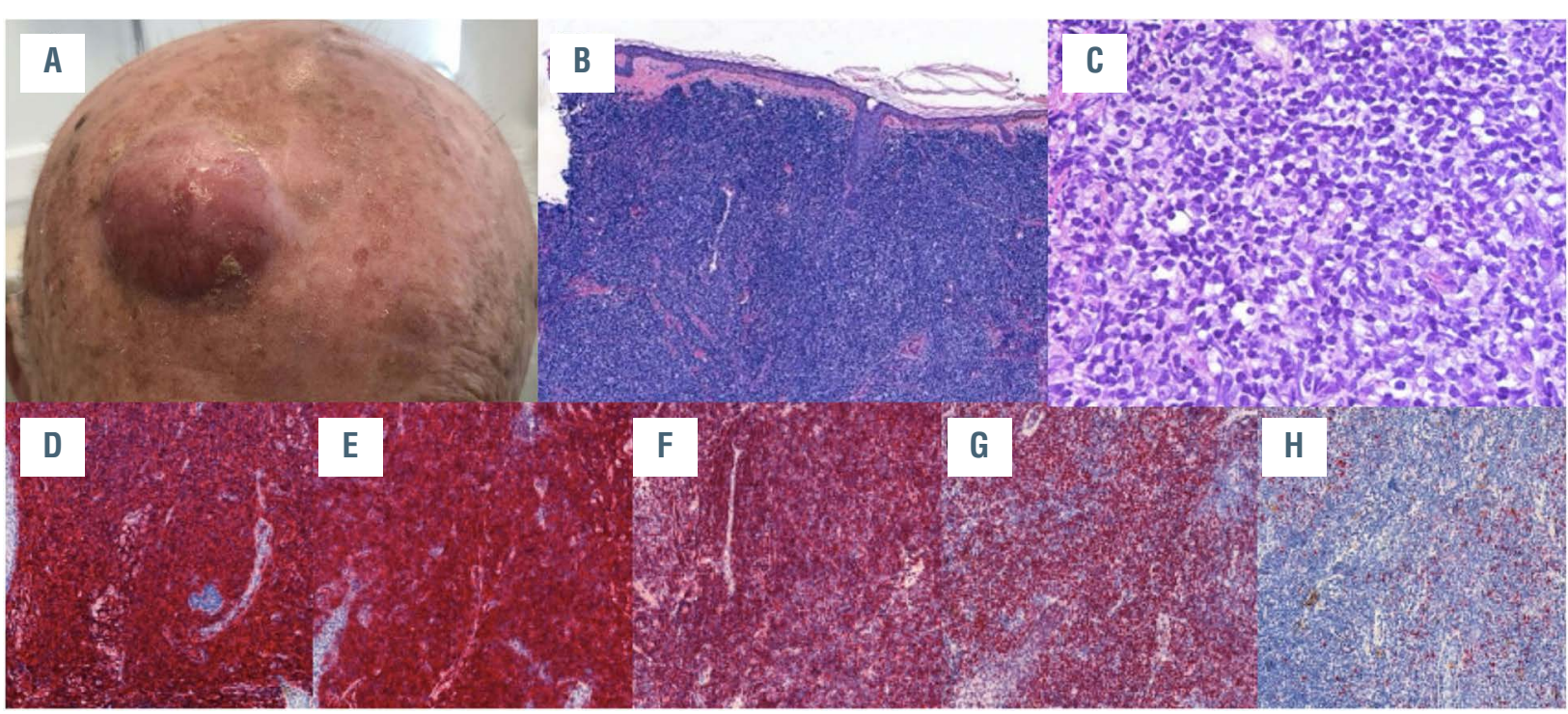

Figure 2. A different presentation of primary cutaneous follicle center lymphoma. (A) A single firm erythematous tumor on the right of the forehead. (B, C) Dense infiltrate of medium-sized centroblasts and immunoblasts in the dermis (hematoxylin \& eosin: [B] 40x, [C] 100x) that are (D) CD10-positive, (E) CD20positive, (F) BCL2-negative on large cells and BCL2-positive on reactive cells, (G) BCL6-positive, and (H) MUM1-negative.

phoma, leg type (PCDLBCL, LT). Combination chemotherapy with rituximab plus cyclophosphamide, doxorubicin, vincristine, and prednisone (R-CHOP) was not initiated due to the patient's poor ejection fraction $(48 \%)$. Instead, given his comorbidities and life expectan$\mathrm{cy}$, he received radiation therapy with complete resolution of the lesion, confirmed with PET scan. Due to his aggressive diagnosis, the patient was closely monitored by oncology without recurrence for 2 years until he died from a cardiac arrest due to his comorbidities.

The three cases presented here highlight the challenge of distinguishing between PCFCL and PCDLBCL-LT, two of the three main subtypes of PCBCL. The first two cases of PCFCL were originally diagnosed as DLBCL without specification. The correct diagnosis of diffuse PCFCL was made after a secondary histological consultation along with clinical correlation. ${ }^{4,5}$ The third case illustrates the fact that a PCDLBCL, LT can present on the scalp.

For the first two cases, the absence of a follicular pattern and diffuse sheets of atypical large cells gave an initial impression of DLBCL while the lack of expression of MUM1 and the presence of a reactive infiltrate clearly pointed to the diagnosis of PCFCL., Although all cases showed a diffuse infiltrate on histology, their histomorphology, immunophenotype, pattern of molecular aberration on FISH analysis, and clinical presentation distinguished the correct diagnosis. This underscores the importance of recognizing PCFCL with diffuse pattern to avoid overcalling DLBCL and the resulting unnecessary aggressive treatment.

Histomorphology should be investigated in detail to arrive at the correct diagnosis among cases of PCBCL. ${ }^{3}$ Although diffuse sheets of cells were seen in all cases, a close evaluation of cellular morphology clearly distinguishes DLBCL from other subtypes. Large cells with multiple mitotic figures and nuclear atypia in PCDLBCL, LT contrast directly with the smaller cells and condensed nuclei seen in PCFCL cases (Figures 1C, 2C, and 3C). ${ }^{6}$

Immunohistochemistry is an essential tool in diagnosing subtypes of PCBCL. MUM-1 positivity precludes PCFCL and must be investigated before making a diagno- sis of any subtype of PCBCL. BCL-2 is not expressed by malignant cells in PCFCL, but it may be present in reactive T cells. ${ }^{3}$ In the second case of the series presented here, BCL-2 was originally called positive but upon further evaluation it was clear that only reactive cells expressed BCL-2. FISH studies can be utilized in cases of PCDLBCL, LT, and we found a positive BCL6 gene rearrangement in our case.

Overall, these cases demonstrate the architectural, histomorphological, and immunohistochemical features that can distinguish PCFCL from PCDLBCL, LT and highlight the diagnostic challenges that arise as a result of overlapping characteristics. The clinical impact of this overlap is most acutely felt in PCDLBCL, LT, because of its more aggressive course and the fact that radiation therapy alone is generally considered inadequate..$^{7,8}$ While there is currently no evidence-based standard of care, most cases of PCDLBCL, LT are treated as systemic DLBCL, with R-CHOP chemoimmunotherapy, often with central nervous system prophylaxis, due to the high risk of central nervous system dissemination. ${ }^{9}$ The addition of radiation therapy to chemoimmunotherapy was found to be important in a recent case series. ${ }^{10}$ Despite historical data showing that the outcomes of patients with PCDLBCL,LT have improved since the introduction of modern chemoimmunotherapy, outcomes remain relatively poor. ${ }^{11}$ In addition, many patients are unfit for chemotherapy, due to age or comorbidities. In the cohort reported by Grange et al. in 2014 about $50 \%$ of the patients were older than 80 years. At the moment, frontline radiation therapy, especially for localized, unifocal disease, is an acceptable option for elderly and frail patients, and some patients, including the third case presented here, have durable responses and long-progression free survival. ${ }^{12,13}$

The clinical and histological findings of B-cell lymphomas can vary widely. The clinical picture in the first case reminds readers that it is possible to have multiple lesions in PCFCL, including multifocal lesions, although it is often thought to present as a solitary lesion. The second case emphasizes that histological and immunohisto- 


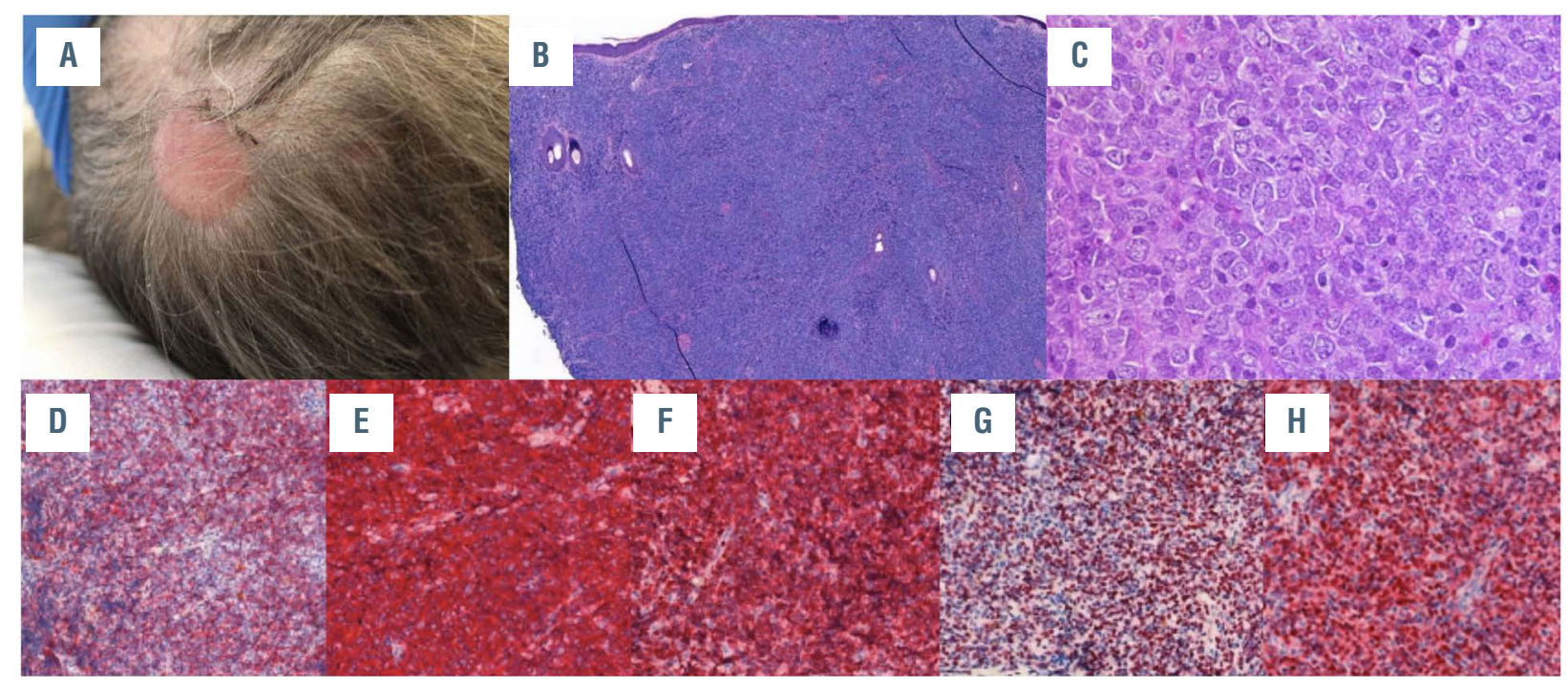

Figure 3. Primary cutaneous diffuse large B-cell lymphoma, leg type is defined by features seen in this figure. (A) A well-defined tumor on the left parietal scalp with $(B, C)$ diffuse proliferation of large polygonal lymphocytes with high mitotic activity, large nuclei, and little cytoplasm (hematoxylin \& eosin: [B] 40x, [C] 100x) that are (D) CD10-positive, (E) CD20-positive, (F) BCL2-positive, (G) BCL6-positive and (H) MUM1-positive.

chemical results must be assessed together, without relying on one over the other. Case three cautions physicians that PCDLBCL, LT can occur elsewhere on the body while having overlapping histological features with PCFCL. Due to the complexity of cutaneous lymphomas, it is imperative for physicians to work together in a multidisciplinary team with dermatology, oncology, dermatopathology and radiation oncology in order to provide the best care for these patients.

Emily Correia, Jisun Cha, ${ }^{1}$ Shalini Krishnasamy, ${ }^{1,2}$ Megan $\mathrm{O}^{\prime}$ Donnell, ${ }^{1}$ Wenyin Shi, ${ }^{3}$ Pierluigi Porcu ${ }^{2}$ and Neda Nikbakht ${ }^{1}$

${ }^{1}$ Department of Dermatology and Cutaneous Biology, Thomas Jefferson University, Philadelphia, PA; ${ }^{2}$ Division of Hematologic Malignancies and HSCT, Department of Medical Oncology, Sidney Kimmel Cancer Center, Thomas Jefferson University, Philadelphia, PA and ${ }^{3}$ Department of Radiation Oncology, Thomas Jefferson University, Philadelphia PA, USA.

Correspondence:

NEDA NIKBAKHT - neda.nikbakht@jefferson.edu

PIERLUIGI PORCU - pierluigi.porcu@jefferson.edu

doi:10.3324/haematol.2021.279992

Received: September 13, 2021.

Accepted: December 2, 2021

Pre-published: December 16, 2021.

Disclosures: no conflicts of interest to disclose

Contributions: EC performed the literature review, wrote the manuscript, and collected illustrations; JC performed histology, helped to write the manuscript, and led discussions of the manuscript; SK and MOD wrote parts of the manuscript; WS wrote part of the manuscript and edited it; PP edited the manuscript; and NN wrote part of the manuscript, edited it, helped with the histology, and led discussions of the manuscript.

Funding: NN is supported by a Skin Cancer Foundation Todd Nagel Memorial Research Grant.

\section{References}

1. Suárez AL, Pulitzer M, Horwitz S, Moskowitz A, Querfeld C, Myskowski PL. Primary cutaneous B-cell lymphomas: part I. Clinical features, diagnosis, and classification. J Am Acad Dermatol. 2013;69(3):329.

2. Mehta-Shah N, Horwitz SM, Ansell S, et al. NCCN guidelines insights: primary cutaneous lymphomas, version 2.2020. J Nat Compr Canc Netw. 2020;18(5):522-536.

3. Willemze R, Cerroni L, Kempf W, et al. The 2018 update of the WHO-EORTC classification for primary cutaneous lymphomas. Blood. 2019;133(16):1703-1714.

4. Malachowski SJ, Sun J, Chen PL, Seminario-Vidal L. Diagnosis and management of cutaneous B-cell lymphomas. Dermatol Clin. 2019;37(4):443-454.

5. Kodama K, Massone C, Chott A, Metze D, Kerl H, Cerroni L. Primary cutaneous large B-cell lymphomas: clinicopathologic features, classification, and prognostic factors in a large series of patients. Blood. 2005;106(7):2491-2497.

6. Felcht M, Klemke CD, Nicolay JP, et al. Primary cutaneous diffuse large B-cell lymphoma, NOS and leg type: Clinical, morphologic and prognostic differences. J Dtsch Dermatol Ges. 2019;17(3):275-285.

7. Senff NJ, Hoefnagel JJ, Neelis KJ, et al. Results of radiotherapy in 153 primary cutaneous B-cell lymphomas classified according to the WHO-EORTC classification. Arch Dermatol. 2007;143(12):15201526.

8. Haverkos B, Tyler K, Gru AA, et al. Primary cutaneous B-cell lymphoma: management and patterns of recurrence at the Multimodality Cutaneous Lymphoma Clinic of The Ohio State University. Oncologist. 2015;20(10):1161-1166.

9. Sundriyal D, Arya L, Srivastava R, Walia M, Sehrawat A. Leptomeningeal relapse in primary ccutaneous DLBCL: implications for a prophylactic CNS therapy. Cancer Rep (Hoboken). 2021;4(1):e1295.

10. Kraft RM, Ansell SM, Villasboas JC, et al. Outcomes in primary cutaneous diffuse large B-cell lymphoma, leg type. J Clin Oncol. 2021;39(15_suppl):e19547.

11. Grange F, Joly P, Barbe C, et al. Improvement of survival in patients with primary cutaneous diffuse large B-cell lymphoma, leg type, in France. JAMA Dermatol. 2014;150(5):535-541.

12. Graham PM, Richardson AS, Schapiro BL, Saunders MD, Stewart DM. Spontaneous regression of primary cutaneous diffuse large Bcell lymphoma, leg type with significant $\mathrm{T}$-cell immune response. JAAD Case Rep. 2018;4(4):305-309.

13. Rodriguez-Pinilla SM, Santonja C, Stewart P, et al. Indolent clinical behaviour of primary cutaneous diffuse large B-cell lymphoma, leg type, with double MYC and BCL6 gene rearrangement. Br J Haematol. 2020;191(3):e83-e86. 